\title{
AIDS and Sexually Transmitted Infections in Africa
}

Oana Streinu-Cercel ${ }^{*}$

Editor

One of the major infectious diseases events of 2011 was the $16^{\text {th }}$ International Conference on AIDS and Sexually Transmitted Infections in Africa (ICASA) that took place at Addis Ababa, Ethiopia, during 4-8 December 2011.

ICASA 2011 covered topics such as: Translating knowledge into practice: country experiences of implementing early infant diagnosis and treatment; Family focused HIV prevention amongst children, with presentations such as: Extent of exclusive infant feeding among HIV positive women in selected PMTCT (preventing mother-to-child transmission) sites in resource poor settings in Nairobi; Traditional birth attendants: a force to reduce mother to child transmission and enhance maternal and newborn child health in Kenya.

Dr. Meskerem Grunitzky-Bekele (Director of the UNAIDS Regional Support Team for West and Central Africa) painted the picture of a 'Journey through 30 years of AIDS - African leadership in the HIV response' and brought news on the status of HIV in Africa, stressing on the fact that not only has antiretroviral coverage in sub-Saharan Africa increased by $20 \%$ between 2009 and 2010, but countries such as Botswana, Namibia and Rwanda have achieved universal access to treatment, defined as a coverage of $80 \%$ or higher. Dr. Grunitzky-Bekele also described the need for further financing, given the

${ }^{*}$ Carol Davila University of Medicine and Pharmacy, 37 Dionisie Lupu Street, Bucharest, 020022; oanastreinucercel@gmail.com

Article downloaded from www.germs.ro

Published on 1 March 2012

(C) GERMS 2012

ISSN $2248-2997$

ISSN - L = 2248 - 2997 impending risk of restricting access to antiretroviral therapy as donor funding decreases.

Dr. Abere Shiferaw (College of Medical Sciences, Haramaya University, Ethiopia) presented the results of a study on the rate of cryptococcal infection and its predictive factors in 256 HIV-infected patients, reporting that $9.77 \%$ of the participants tested positive for Cryptococcus neoformans. The study also found that screening for cryptococcal antigenemia revealed relatively high rates of asymptomatic cryptococcosis in subSaharan Africa. Dr. Shiferaw recommended that HIV patients with CD4 counts $<200$ cells/cmm be routinely screened for asymptomatic cryptococcal infection, in order to initiate appropriate preemptive antifungal therapy early and prevent immune reconstitution inflammatory syndrome due to cryptococcosis.

ICASA also hosted a large number of interesting workshops such as: Integrating planning, monitoring and evaluation to increase effectiveness of HIV/AIDS related interventions: the Logframe approach; Quantitative methods for evaluating behavioral HIV prevention interventions; Laboratory strengthening through the WHO Stepwise Laboratory Improvement Process Towards Accreditation (SLIPTA).

What have we learned from ICASA 2011? We have learned that the number of new HIV infections in sub-Saharan Africa has dropped by more than 26\% since 1997 (Dr. GrunitzkyBekele); that the national antenatal survey showed a 30\% seroprevalence of infection for 2010 (Dr. Pascal Bessong, Department of Microbiology, University of Venda, South Africa) and much, much more.

For further details on the conference, visit the conference website at http://www.icasa2011addis.org/ 